\title{
Criminologie
}

\section{Hommage à Denis Szabo}

\section{Pierrette Trudeau-LeBlanc}

Volume 19, numéro 1, 1986

Politiques et pratiques pénales. 25 ans de réflexion et d'action

URI : https://id.erudit.org/iderudit/017223ar

DOI : https://doi.org/10.7202/017223ar

Aller au sommaire du numéro

Éditeur(s)

Les Presses de l'Université de Montréal

ISSN

0316-0041 (imprimé)

1492-1367 (numérique)

Découvrir la revue

Citer ce document

Trudeau-LeBlanc, P. (1986). Hommage à Denis Szabo. Criminologie, 19(1), 7-9. https://doi.org/10.7202/017223ar d'utilisation que vous pouvez consulter en ligne.

https://apropos.erudit.org/fr/usagers/politique-dutilisation/ 
La criminologie a plus de cent cinquante ans d'existence. Comme discipline autonome, on l'enseigne au Québec depuis 25 ans et la profession de criminologue est encore plus jeune. Personne n'ignore le nom de l'homme qui, en 1960, a fondé l'École de criminologie de l'Université de Montréal, Denis Szabo.

Autant dans les milieux des sciences exactes, de la médecine que dans les milieux juridiques, que vous rencontiez un policier, un journaliste ou un homme de la rue, de Paris à Moscou et de Caracas à Abidjan, chacun associe le nom du docteur Szabo à la criminologie. Quant aux criminologues du Québec, qu'ils partagent ou non sa philosophie, ses orientations, ou ses prises de position, ils reconnaissent tous en Denis Szabo, l'un des grands concepteurs de leur discipline sous sa forme actuelle. Certains voient même en lui «le père de la criminologie moderne».

Hongrois d'origine, issu d'une famille de militaires de Budapest et en dissidence vis-à-vis des choix politiques qui s'offraient dans son pays, Denis Szabo a poursuivi ses études en Belgique et en France. En dépit de nombreuses difficultés d'ordre matériel et linguistique, il obtient en 1956, un doctorat en sciences sociales et politiques de l'Université de Louvain et en 1958, un diplôme de l'École pratique des hautes études de Paris. Au cours de ces mêmes années, il est chargé de cours (sociologie) aux Universités catholique de Paris et de Lyon et enseigne à l'Université catholique de Louvain. Déjà, il se distingue par ses qualités professionnelles, ses recherches et son intérêt pour la criminologie.

En 1958, à l'invitation de M. l'abbé Norbert Lacoste, professeur de sociologie à l'Université de Montréal et devant l'attrait des horizons nouveaux et prometteurs, il immigre au Québec où il devient à son tour professeur de sociologie (sociologie de la déviance et méthodes de recherche) à l'Université de Montréal. C'est là que, toujours à l'affut des besoins de la société, attentif au contexte socio-politique de l'époque et très confiant dans le développement de la sociologie criminelle, il fonde, en 1960, le

* M.Sc. (Criminologie), criminologue praticienne. 
Département de criminologie, avec l'appui des autorités en place, favorables aux initiatives nouvelles. Le Département, qui, dans l'esprit de son fondateur, se voulait au carrefour des sciences humaines offrait un enseignement multidisciplinaire destiné à former des chercheurs et des praticiens.

Pour le docteur Szabo, la criminologie est d'abord une science appliquée, elle ne se limite pas à la recherche, et elle doit s'étendre à toutes les sphères du problème criminel. À son instigation, en 1960 , sera créée la Société de criminologie du Québec dont il sera le premier secrétaire général. Par ses congrès, ses colloques de recherche et ses publications, la Société a d'ailleurs joué un rôle de premier plan auprès du public et des gouvernements dans l'orientation des politiques pénales du Québec et du Canada.

En 1968, toujours préoccupé de diffuser les connaissances et les travaux québécois et canadiens, Szabo n'a pas hésité à mettre sur pied une revue, Acta Criminologica, qui prendra plus tard le nom de Criminologie, et dont il est encore le directeur.

M. Szabo a toujours été un habile «commerçant» d'idées. N'a-t-il pas «importé» la criminologie au Canada? À ce propos, il est amusant de se rappeler ses propres paroles lancées avec humour, à la veille d'un de ses nombreux départs pour l'étranger, alors qu'il croulait littéralement sous les abondantes publications scientifiques qu'il se proposait de distribuer lors de rencontres internationales : "Voyez, je suis un véritable colporteur d'idées qui part à l'assaut des lointaines contrées pour y porter la Bonne Nouvelle !» C'est dans cette veine, qu'en 1967, il a conçu le XVII ${ }^{\mathrm{e}}$ Cours international de criminologie qui s'est tenu à Montréal. Les trois thèmes qui y furent abordés, la personnalité criminelle, l'administration de la justice et la prévention, sont restés depuis lors des lignes directrices pour la recherche et la pratique. De nombreux échanges européens et nord-américains prirent d'ailleurs naissance à cette époque. Et se poursuivent encore aujourd'hui alimentés par de nombreuses autres rencontres comme, par exemple, le IX ${ }^{\mathrm{e}}$ Congrès international de Vienne que M. Szabo a organisé en 1983 autour de la relation de la criminologie avec les politiques et pratiques.

Quand en 1969, Denis Szabo a fondé le Centre international de criminologie comparée, c'était toujours avec l'intention de promouvoir la collaboration internationale, dans l'enseignement, la recherche et la diffusion de la criminologie. Ce centre constitue 
toujours le noyau d'un réseau de chercheurs dont les préoccupations scientifiques sont centrées sur leur milieu tout en demeurant ouvertes sur le monde.

Très dynamique, le docteur Szabo est membre et animateur de nombreuses associations internationales et il dirige ou fait partie de plusieurs comités de rédaction de périodiques scientifiques. Conseiller et expert auprès d'organismes nationaux et internationaux en matière criminelle, qu'il peut faire bénéficier de son grand esprit de synthèse de même que de ses vastes connaissances des cultures et des époques. Il a reçu, entre autres, deux doctorats honoris causa des universités de Vienne et de Budapest. De 1978 à 1985, il fut le premier président canadien de la Société internationale de criminologie qui, comme on sait, constitue l'une des tribunes par excellence des échanges scientifiques avec l'Europe.

Communicateur hors pair, toujours enthousiaste et souvent enthousiasmant, Denis' Szabo poursuit sa carrière en préparant de nouveaux ouvrages qui s'ajouteront à la liste déjà longue de ses livres et de ses articles. Homme d'idées et d'action, il demeure l'initiateur de la criminologie canadienne et le promoteur des échanges internationaux sur le phénomène criminel et les politiques pénales.

Les réalisations et les publications de $M$. Szabo sont trop nombreuses pour qu'on puisse en dresser ici un bilan exhaustif qui d'ailleurs ne pourrait êtro que partiel, à en juger par ses multiples projets en gestation ou en voie d'accomplissement. Mais celles que nous avons mentionnées suffisent à décrire son «tempérament intellectuel», son «mode de penser». Denis Szabo, selon ses propres termes, se range parmi les «esprits qui, comme le renard d'Archiloque, sont attentifs à plusieurs choses simultanément». Il est de ceux qui «suivent de nombreuses pistes à la fois, sans liens apparents, si ce n'est qu'une base intuitive, une sorte d'exigence morale ou esthétique» (Szabo, D. la Criminologie. Bilan et perspectives. Mélanges offerts à Jean Pinatel, Paris, Pédone, 1980, p. 32). 